\title{
WHITNEY STRATIFIED CHAINS AND COCHAINS
}

BY

\section{R. MARK GORESKY}

\begin{abstract}
ABSTRACr. This paper contains the technical constructions necessary for a "geometric cycle" definition of cohomology and homology in the context of Whitney stratifications. Cup and cap products are interpreted as the transverse intersection of geometric cocycles and cycles.
\end{abstract}

1. Introduction. The purpose of this paper is to develop a geometric theory of cycles, cocycles and intersections within the "category" of Whitney stratifications. Whitney stratifications form an important class of spaces because: (1) they arise naturally in the study of algebraic, analytic and subanalytic varieties, polyhedra, and singularities of smooth mappings; (2) they are preserved under transversal intersections and the "perturbation" techniques of differential topology can be used to make them transverse; (3) they determine currents by integration [22]; and (4) their local topological structure has been explicitly described by Thom [20] and Mather [13].

The main theorems are 4.7 and 6.2:

Let $X$ be a compact Whitney stratified set. The cobordism group of embedded normally oriented Whitney stratified subsets which are "transverse to the singularities" of $X$ coincides with the integral cohomology of $X$, and transverse intersection of these geometric cocycles corresponds to the cup product.

This theorem reflects a principle (popularized by Dennis Sullivan) that "cup product is transverse intersection of cocycles with normal geometry", although it is difficult to find a correctly stated version of this principle in the literature. An attempt at a geometric theory of cocycles was made by Whitney [25] who did not have the techniques needed to make his results rigorous. Using different methods, Buoncristiano, Rourke, and Sanderson [1] have been more successful in the P.L. context: one must observe that in the case of ordinary cohomology, the Mock bundles can be taken to be embedded subsets. It is also well known that the generalized "dual cells" in a simplicial complex form geometric representatives of cohomology classes, although the cup product is not so easily interpreted as an intersection since the dual cells are not always transverse to each other.

In 8.2 we identify a "universal" geometric cocycle in a stratification of the Eilenberg-Mac Lane space $K(Z, n)$, thus answering a question of Thom [21].

This paper is partly concerned with addressing the technical difficulties involved in manipulating Whitney stratified sets. For example, the "push-forward problem"

Received by the editors January 23, 1980 and, in revised form, September 23, 1980.

1980 Mathematics Subject Classification. Primary 57R95, 57N80; Secondary 55N20, 55M05. 
is still open: to find natural sufficient conditions on a Whitney stratified subset $X$ of a smooth compact manifold $M$, such that for almost every smooth mapping $f$ : $M \rightarrow N$ the image $f(X)$ has a Whitney stratification which is compatible with that of $X$.

Our main technical theorem appears in the appendix, which is the heart of the proof of the main theorems 3.4 and 4.7. It is not known at present whether every Whitney stratified set admits a triangulation such that every simplex satisfies the Whitney conditions. We prove that every Whitney stratified set can be deformed to one which has a Whitney cellulation, and the whole deformation can be made to satisfy the Whitney conditions.

An essential step in the proof of the main theorem 4.7 is Theorem 3.4: The cobordism group based on (embedded) compact oriented Whitney stratified subsets of a smooth manifold $M$ coincides with the integral homology of $M$. This notion of "geometric chain" resembles Poincarés original definition of chain (which he was forced to modify in the interests of rigor). Geometric cycle theorems exist in many other categories: in the P.L. [1], the real analytic [9] and the subanalytic [10] categories, and in the context of currents [3], so this result is not surprising. However there are situations where naturally occurring geometric cycles may have a Whitney stratification even though they do not have a natural P.L. or subanalytic structure, for example in the study of generic singularities of smooth maps (3.7). It is also a long standing conjecture than any minimal "surface" representing a given homology class in a Riemannian manifold, has a Whitney stratification. Theorem 3.4 also provides a good context in which to ask questions like the following: what is the maximum codimension of the singularity set of any geometric cycle which represents a given homology class in a given manifold? (This refines the question answered by Thom [19] on the representability of homology classes by submanifolds.)

In $\$ 9$ we consider related " $\pi$-fibre" Morse functions on a stratified set $X$ and show that $X$ has the homotopy type of a CW complex with one cell of dimension $\lambda$ for each critical point of index $\lambda$.

I am very happy to thank Bob MacPherson for his advice and encouragement. He conjectured many of the results in this paper, helped to clarify some of the technical details, and persuaded me to write them down. I have also profited from useful conversations with Clint McCrory, Richard Porter, and Dennis Sullivan.

2. Whitney stratifications. In this section we review the main results of Thom and Mather [5], [13], [14], [20] and fix notation. All manifolds are $C^{\infty}$.

Definition. A tubular neighborhood $T_{N}$ of a submanifold $N$ of a manifold $M$ consists of (a) a choice of Riemannian metric on the fibres of the normal bundle $\pi$ : $E \rightarrow N$ and (b) a smooth embedding $\phi: E_{\delta} \rightarrow M$ where $E_{\delta}=\{v \in E \mid\langle v, v\rangle\langle\delta\}$, such that $T_{N}=\phi\left(E_{\delta}\right)$ is a neighborhood of $N$ in $M$ and $\phi$ takes the zero section of $E$ identically to $N$. This determines a tubular "distance function" $\rho_{N}(x)=$ $\left\langle\phi^{-1}(x), \phi^{-1}(x)\right\rangle$ and tubular "projection" $\pi_{N}=\phi \circ \pi \circ \phi^{-1}: T_{N} \rightarrow N$. Let $T_{N}(\varepsilon)=$ $\left\{x \in T_{N} \mid \rho(x)<\varepsilon\right\}$ and $S_{N}(\varepsilon)=\left\{x \in T_{N} \mid \rho(x)=\varepsilon\right\}$, for $\varepsilon<\delta$.

A Whitney stratification of a closed subset $X$ of a smooth manifold $M$ is a filtration of $X$ by closed subsets $X_{0} \subset X_{1} \subset \cdots \subset X_{n}$ such that each $X_{i}-X_{i-1}$ is 
a (possibly empty) locally finite union of $i$-dimensional submanifolds of $M$ (the components of which are called the strata of dimension $i$ ), such that each pair of strata satisfies Whitney's condition $B$ [4], [11], [18]. It follows that if $S$ and $T$ are strata and $S \cap \bar{T} \neq \varnothing$ then $S \subset \bar{T}$ and we write $S<T$. The subset $X$, together with this structure is called a Whitney object; $X_{i}$ is called its $i$-skeleton. Each such Whitney object $X \subset M$ admits a system of control data which consists of a tubular neighborhood $T_{A}$ (in $M$ ) of each stratum $A$, with the following properties:

(1) The map $\left(\pi_{A}, \rho_{A}\right): T_{A} \rightarrow A \times[0, \varepsilon)$ has surjective differential when restricted to any stratum $B>A$.

(2) For each $B>A$ the relations $\pi_{A} \circ \pi_{B}=\pi_{A}$, and $\rho_{A} \circ \pi_{B}=\rho_{A}$ hold whenever both sides of the equation are defined.

(3) For all $\varepsilon$ sufficiently small, the sphere bundles $S_{A}(\varepsilon)$ are multi-transverse, which means that if $A_{1}, A_{2}, \ldots, A_{k}$ is any collection of strata, and $B_{1}, B_{2}, \ldots, B_{l}$ is any disjoint collection, that the intersection $S_{A_{1}}(\varepsilon) \cap \cdots \cap S_{A_{k}}(\varepsilon)$ is transverse to the intersection $S_{B_{1}}(\varepsilon) \cap \cdots \cap S_{B_{l}}(\varepsilon)$, and is also transverse to any other stratum $C$ of $X$.

A Whitney substratified object $W \subset X$ is a closed subset with a Whitney stratification (this makes sense since $W \subset M)$ such that each stratum of $W$ is contained in a single stratum of $X$. ( $W$ is not necessarily a union of strata of $X$.)

3. Geometric chains and homology. In this section we fix a Whitney object $X$ (in some manifold) and construct a homology theory whose cycles are embedded oriented Whitney objects in $X$ and whose homologies are embedded oriented cobordisms in $X \times[0,1]$. The main result (Theorem 3.4) identifies this homology theory with the ordinary integral homology of $X$, in the case that $X$ is a manifold.

3.1. Definition. $A$ geometric $k$ chain $\xi$ in $X$ consists of a compact $k$ dimensional Whitney substratified object $|\xi| \subset X$ (called the support of $\xi$ ) together with an orientation of $|\xi|$ which is a choice of an orientation and multiplicity of each $k$-dimensional stratum (however we identify a given orientation and multiplicity with the opposite orientation and negative multiplicity, and allow nonorientable components provided their multiplicity is 0 ). The set of orientations of $|\xi|$ is just the group $H_{k}\left(|\xi|,|\xi|_{k-1}\right)$.

The reduction of a geometric $k$ chain $\xi$ is the geometric chain whose support is the closure of the union of all components of $|\xi|-|\xi|_{k-1}$ which have been assigned a nonzero multiplicity. We shall identify a geometric chain with its reduction.

The boundary $\partial \xi$ of a geometric $k$ chain $\xi$ is the (reduction of the) geometric $k-1$ chain whose support is $|\xi|_{k-1}$ and whose orientation is induced from $H_{k}\left(|\xi|,|\xi|_{k-1}\right) \rightarrow H_{k-1}\left(|\xi|_{k-1}\right) \rightarrow H_{k-1}\left(|\xi|_{k-1},|\xi|_{k-2}\right)$.

A geometric $k$-cycle is a geometric $k$ chain $\xi$ such that $\partial \xi=0$. In this case the orientation of $\xi$ pulls back to a unique fundamental class $\mu_{\xi} \in H_{k}(|\xi|)$. We shall say $\xi$ represents the homology class $[\xi]=i_{*} \mu_{\xi} \in H_{k}(X)$ where $i:|\xi| \rightarrow X$ is the inclusion.

3.2. Geometric interpretation of the boundary. An orientation of $\xi$ is equivalent to a continuous choice of orientation and multiplicity of the tangent spaces $T_{x} S$ for each $k$-dimensional stratum $S$. The boundary orientation and multiplicity on the 
tangent space $T_{y} R$ of any $k-1$ dimensional stratum $R$ is determined geometrically as follows: consider a small $\varepsilon$-ball $B$ centered at $y \in R$ and let $S_{1}, \ldots, S_{p}$ denote the connected components of $B \cap\left(|\xi|-|\xi|_{k-1}\right)$. For each $i$, let $x_{i} \in S_{i}$ be chosen so that the oriented secant line $l_{i}=\overrightarrow{y x}_{i}$ is almost orthogonal to $T_{y} R$ and is almost contained in $T_{x_{i}} S_{i}$, and such that $T_{y} R$ is almost contained in $T_{x_{i}} S_{i}$. Then orthogonally projecting into $T_{x_{i}} S_{i}$ gives an isomorphism $T_{y} R \oplus l_{i} \rightarrow T_{x_{i}} S_{i}$. There is a unique orientation $\theta_{i}$ of $T_{y} R$ such that the product orientation on $T_{y} R \oplus l_{i}$ agrees with the given orientation of $T_{x_{i}} S_{i}$. Finally, if $m_{i}$ denotes the multiplicity of $S_{i}$, the sum $\sum m_{i} \theta_{i}$ is the boundary orientation and multiplicity of $T_{y} R$.

3.3. Definition. Two geometric $k$-cycles $\xi_{0}$ and $\xi_{1}$ in $X$ are cobordant if there is a geometric $k+1$ chain $\eta$ in $X \times \mathbf{R}$, and some $\varepsilon>0$ such that

(a) $|\eta| \subset X \times[0,1]$

(b) $|\eta| \cap X \times[0, \varepsilon)=\left|\xi_{0}\right| \times[0, \varepsilon)$,

(c) $|\eta| \cap X \times(1-\varepsilon, 1]=\left|\xi_{1}\right| \times(1-\varepsilon, 1]$,

(d) $\partial \eta=\xi_{1} \times\{1\}-\xi_{0} \times\{0\}$ (modulo reduction).

Cobordism is an equivalence relation. Let $W H_{k}(X)$ denote the set of cobordism classes of geometric $k$-cycles in $X$. Cobordant cycles clearly represent the same homology class in $X$, so we obtain a representation map $R: W H_{k}(X) \rightarrow H_{k}(X)$.

\subsection{TheOrem. If $X$ is a manifold then $R$ is a bijection.}

Proof. Choose a smooth triangulation of $X$. The closure of any union of simplices is Whitney stratified by its decomposition into simplices. Any homology class in $H_{k}(X)$ has a representative as a simplicial cycle which is therefore also a geometric cycle. Thus $R$ is surjective.

To show $R$ is injective, suppose $\xi_{0}$ and $\xi_{1}$ are geometric $k$ cycles which represent the same class in $H_{k}(X)$. According to appendix A.3, $\xi_{0}$ is cobordant to a geometric $k$-cycle $\xi_{0}^{\prime}$ with conical singularities. By A.2 there is a regular cell decomposition of $X \times\{0\}$ so that $\left|\xi_{0}\right| \times\{0\}$ is a union of cells and so that the closure of each cell satisfies the Whitney conditions. Similarly $\xi_{1}$ is cobordant to a geometric cycle $\xi_{1}^{\prime}$ with conical singularities, such that $\left|\xi_{1}^{\prime}\right| \times\{1\}$ is a union of cells of a Whitney cellulation of $X \times\{1\}$. These cellulations extend to a Whitney cellulation of $X \times[0,1]$ by applying A.2 again. Therefore $\xi_{0}^{\prime}$ and $\xi_{1}^{\prime}$ are homologous cellular cycles in this cellulation of $X \times[0,1]$ and there is a cellular chain $\eta$ such that $\partial \eta=\xi_{1}^{\prime} \times\{1\}-\xi_{0}^{\prime} \times\{0\}$. This chain $\eta$ determines the desired cobordism.

3.5. Remark on relative homology. If $X$ is a manifold with collared boundary (or even a manifold with collared corners), define a relative geometric cycle $\xi$ to be a compact oriented Whitney object $|\xi| \subset X$ which respects the collaring, such that the support of $\partial \xi$ is contained in $\partial X$. Relative cobordisms can be similarly defined. Relativizing the proof of 3.4 (using the relative version of appendix A.2) we obtain a bijection between the relative geometric $k$ cycles, $W H_{k}(X, \partial X)$ and the relative homology group $H_{k}(X, \partial X)$.

Theorem 3.4 may even be true if $X$ is any Whitney object, but a proof would apparently involve an extension of the techniques in the Appendix. We can at least see that if $X$ is a subanalytic set then $W H_{k}(X) \rightarrow H_{k}(X)$ is surjective since every homology class in $X$ has a subanalytic representative. 
3.6. Intersections in homology. Two Whitney objects $X$ and $Y$ in a smooth manifold $M$ are transverse if each stratum of $X$ is transverse to each stratum of $Y$. In this case $X \cap Y$ has a Whitney stratification whose strata are (the components of) manifolds of the form $A \cap B$ where $A$ is a stratum of $X$ and $B$ is a stratum of $Y$ [13].

THEOREM. Suppose $M$ is an oriented $n$-manifold and let $\xi$ be a geometric $i$-cycle and let $\eta$ be a geometric j-cycle in $M$. Then there is a diffeomorphism $\theta: M \rightarrow M$ arbitrarily close to the identity such that $\theta(|\xi|)$ is transverse to $|\eta|$. If $\xi^{\prime}$ denotes this "perturbed" cycle then $\left[\xi^{\prime}\right]=[\xi]$ and the product orientation on $\left|\xi^{\prime}\right| \cap|\eta|$ gives an $i+j-n$ dimensional intersection cycle $\xi^{\prime} \cap \eta$ (see 6.1). Furthermore $\left[\xi^{\prime} \cap \eta\right]$ repre-

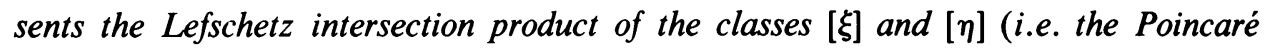
dual to the cup product in the cohomology with compact supports of M.)

Proof. This is a special case of Theorem 6.2.

3.7. EXAMPLES. (i) Any compact complex analytic subvariety $W$ of an analytic variety $X$ is a geometric cycle and therefore represents a homology class in $X . W$ has a Whitney stratification consisting of complex analytic strata so the orientation is canonical. The cycle condition is automatic since there are no codimension one strata.

(ii) Suppose $M^{n}$ is a smooth compact submanifold of $\mathbf{R}^{N}$ and let $\pi: \mathbf{R}^{N} \rightarrow \mathbf{R}^{P}$ denote the projection to the first $p$ coordinates. If the Gauss map $g: M \rightarrow G_{n}\left(\mathbf{R}^{N}\right)$ is transverse to the appropriate Schubert variety then the singularity set $\Sigma^{1}=\{x \in$ $\left.M \mid \operatorname{corank}\left(d \pi \mid T_{x} M\right) \geqslant 1\right\}$ has a Whitney stratification and becomes a $(\bmod 2)$ geometric cycle which represents the Poincare dual of the Stiefel-Whitney class $W^{n-p+1}(M)$. More generally, the singularity set of a generic (real or complex) vector bundle map (over a smooth manifold) carries the structure of a geometric cycle whose homology class is considered in [12].

4. Geometric cochains and cohomology. In this section, $X$ will denote a fixed $n$-dimensional Whitney object (in some manifold $M$ ) with a fixed system of control data. We will construct a cohomology theory on $X$ whose cycles are codimension $k$ normally oriented Whitney objects embedded in $X$ which satisfy the $\pi$-fibre condition, and whose relations are $\pi$-fibre cobordisms of codimension $k$, embedded in $X \times[0,1]$. The main result (4.7) identifies this geometric cohomology group with the ordinary cohomology group $H^{k}(X)$.

4.1. The $\pi$-fibre condition. A substratified object $W \subset X$ is said to satisfy the $\pi$-fibre condition (with respect to the chosen system of control data on $X$ ) if for each stratum $S$ of $X$ there is an $\varepsilon>0$ such that

$$
W \cap T_{S}(\varepsilon)=\pi_{S}^{-1}(W \cap S) \cap T_{S}(\varepsilon) \cap X .
$$

We also assume this identification is compatible with the stratifications of $W$ and of $\pi_{S}^{-1}(W \cap S) \cap X$. (From now on, we will surpress the $\varepsilon$.)

In this case, $W$ has a costratification defined as follows: If $A$ is a stratum of $W$ which is contained in a stratum $S$ of $X$, define the intrinsic codimension of $A$ to be $\operatorname{dim}(S)-\operatorname{dim}(A)$ and the intrinsic normal bundle of $A$ to be the normal bundle of 
$A$ in $S$. A codimension $k$ costratum of $W$ is defined to be (any connected component of) the union of all the strata of $W$ which have intrinsic codimension equal to $k$. Define the $p$-coskeleton $W^{(p)}$ to be the union of all costrata which have codimension $p$ or more. The $W^{(p)}$ form a filtration of $W$ by (closed) $\pi$-fibre substratified objects.

Each codimension $k$ costratum $D$ of $W$ has a normal vector bundle $E_{D}$ and a tubular neighborhood $T_{D}$ in $X$, which is the image of an embedding $\phi: E_{D} \rightarrow X$, as follows:

$E_{D}$ is the union of the intrinsic normal bundles of each stratum of $D$. These fit together in a locally trivial bundle over $D$ because $D$ satisfies the $\pi$-fibre condition and in fact, for each stratum $S$ of $X, E_{D} \mid\left(D \cap T_{S}\right)=\pi_{S}^{*}\left(E_{D} \mid D \cap S\right)$. Similarly $T_{D}$ is the union of intrinsic tubular neighborhoods of the strata of $D$. It can be constructed by ascending induction on the dimension of the strata of $D$ by demanding that, for any $v \in E_{D} \mid\left(D \cap T_{S}\right), \pi_{S}(\phi(v))=\phi\left(d \pi_{S}(v)\right)$. Here $d \pi_{S}$ denotes the vector bundle map $E_{D}\left|\left(D \cap T_{S}\right) \rightarrow E_{D}\right|(D \cap S)$ which covers the projection $\pi_{S}: D \cap T_{S} \rightarrow D \cap S$.

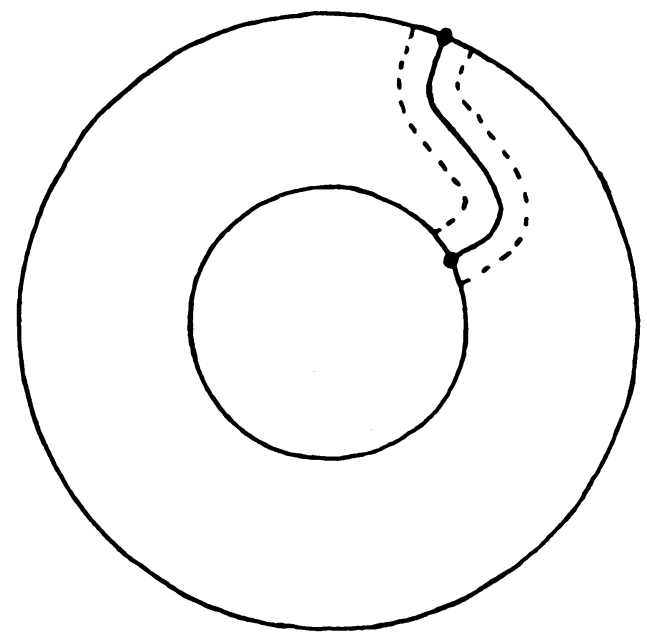

Tubular neighborhood of a $\pi$-fibre cocycle in the annulus

4.2. Coorientations. Let $D$ be a codimension $k$ costratum of a $\pi$-fibre substratified object $W \subset X$. Let $E_{D}$ be the normal bundle to $D$ with fibre inclusion $j_{p}: E_{p} \subset E_{D}$. A coorientation of $D$ is a locally constant choice of orientation (i.e. generator $\theta_{p} \in H_{k}\left(E_{p}, E_{p}-p\right)$ ) and multiplicity $m_{p} \in \mathbf{Z}$ of the fibres of $E_{D}$, (however we identify a given orientation and multiplicity with the reverse orientation and negative multiplicity). Each coorientation corresponds to a unique element $\mu_{D} \in$ $H^{k}\left(E_{D}, E_{D}-D\right)$ which is determined by the condition $\left\langle j_{p}^{*}\left(\mu_{D}\right), \theta_{p}\right\rangle=m_{p}$ for each point $p \in D$.

4.3. Definition. $A$ codimension $k$ geometric cochain $\theta$ in $X$ consists of a $\pi$-fibre substratified object $|\theta| \subset X$ (called the support of $\theta$ ) which has a costratification such that all costrata have codimension $\geqslant k$, together with a coorientation of each 
codimension $k$ costratum. It follows from the existence of tubular neighborhoods (4.1) and excision, that adding the coorientations $\mu_{D}$ over all the codimension $k$ costrata $D$ gives a class $\mu_{\theta}$ in

$$
\begin{aligned}
\oplus H^{k}\left(T_{D}, T_{D}-D\right) & \cong \oplus H^{k}(X-(\bar{D}-D), X-\bar{D}) \\
& \cong H^{k}\left(X-|\theta|^{k+1}, X-|\theta|\right) .
\end{aligned}
$$

(Here, $|\theta|^{k+1}$ denotes the $k+1$-coskeleton of $|\theta|$.)

The reduction of $\theta$ is the geometric cochain whose support is the closure of the union of all costrata which have been assigned a nonzero multiplicity. We shall identify cochains which have the same reduction.

4.4. The coboundary $\delta \theta$ of a codimension $k$ geometric cochain $\theta$ is the (reduction of the) geometric cochain whose support is the coskeleton $|\delta \theta|=|\theta|^{(k+1)}$ and whose coorientation is the image of $\mu_{\theta}$ under the map $\delta$ in the following diagram:

$$
\begin{array}{cc}
H^{k}\left(X, X-|\theta|^{k+1}\right) \rightarrow H^{k}(X, X-|\theta|) \rightarrow H^{k}\left(X-|\theta|^{k+1}, X-|\theta|\right) \rightarrow & H^{k+1}\left(X, X-|\theta|^{k+2}\right) \\
\downarrow & H^{k+1}\left(X, X-|\theta|^{k+1}\right) \\
H^{k}(X) & H^{k+1}\left(X-|\theta|^{k+2}, X-|\theta|^{k+1}\right)
\end{array}
$$

(The coboundary coorientation and multiplicities on the normal bundles of the codimension $k+1$ costrata may also be defined locally using inward pointing normal vectors as in 3.2.)

The geometric cochain $\theta$ is a geometric cocycle if $\delta \theta=0$. The following lemma implies that $\mu_{\theta}$ lifts to a unique class in $H^{k}(X, X-|\theta|)$. Its image in $H^{k}(X)$ is denoted $[\theta]$.

4.5. Lemma. $H^{k}\left(X, X-|\theta|^{k+1}\right)=H^{k+1}\left(X, X-|\theta|^{k+2}\right)=0$.

Proof. We claim that whenever $W \subset X$ is a $\pi$-fibre Whitney object of codimension $c$ and if $p<c$ then $H^{p}(X, X-W)=0$. The case $c=\operatorname{dim}(X)=n$ is clear since $(X, X-W)$ is homotopic to a wedge of $n$-spheres. By decreasing induction suppose $\operatorname{codim}(W)=k$, so $H^{p}\left(X, X-W^{(k+1)}\right)=0$ whenever $p<k+1$. Then it follows from the cohomology sequence of the triple $\left(X, X-W^{(k+1)}, X-W\right)$ that $H^{p}(X, X-W) \cong H^{p}\left(X-W^{(k+1)}, X-W\right)$ for $p<k$. By excision this equals $H^{p}\left(E_{S}, E_{S}-S\right)$ where $E_{S}$ is the normal bundle of the costratum $S=W-$ $W^{(k+1)}$. However the Thom isomorphism gives $H^{p}\left(E_{S}, E_{S}-S\right)=0$ provided $p<k$.

4.6. Two codimension $k$ geometric cocycles $\theta_{0}$ and $\theta_{1}$ in $X$ are cobordant if there is a ( $\pi$-fibre) codimension $k$ geometric cochain $\eta \subset X \times \mathbf{R}$, and some $\varepsilon>0$ such that

(a) $|\eta| \subset X \times[0,1]$

(b) $|\eta| \cap X \times[0, \varepsilon)=\left|\theta_{0}\right| \times[0, \varepsilon)$,

(c) $|\eta| \cap X \times(1-\varepsilon, 1]=\left|\theta_{1}\right| \times(1-\varepsilon, 1]$,

(d) $\delta \eta=\theta_{1} \times\{1\}-\theta_{0} \times\{0\}$,

Cobordism is an equivalence relation. Define the geometric cohomology set $W H^{k}(X)$ to be the set of cobordism classes of codimension $k$ geometric cocycles. It 
is easy to see from 4.4 that cobordant cocycles represent the same cohomology class and we obtain the representation map

$$
R: W H^{k}(X) \rightarrow H^{k}(X) .
$$

\subsection{THEOREM. $R$ is a bijection.}

The proof of this theorem for compact $X$ is in $\S 7$. In the noncompact case one must use Borel-Moore homology throughout.

4.8. Remark. If $X$ is an "abstract stratified space" [13], [20] define geometric cochains in $X$ as in 4.3 to be cooriented $\pi$-fibre substratified objects which satisfy the Whitney conditions within each stratum of $X$. Then Theorem 4.7 holds for such an $X$ because it is a fact (not proven here) that $X$ admits a Whitney embedding $\tilde{X}$ in some Euclidean space which identifies geometric cochains in $X$ with geometric cochains in $\tilde{X}$.

4.9. Relative cohomology. If $X$ is a stratified space and $A$ is a closed union of strata then $X / A$ inherits a stratification. Cocycles of codimension $k \geqslant 1$ may not intersect the singular point $A / A \in X / A$ and the same applies to cobordisms. Thus $H^{k}(X, A)$ is given by codimension $k$ geometric cocycles in $X$ with support in $X-A$, modulo cobordisms in $X \times[0,1]$ with support in $(X-A) \times[0,1]$. This is even true when $k=0$ since $H^{0}(X, A)$ is the reduced cohomology of $X / A$.

4.10. Relation to intersection homology. If $X$ is an $n$ dimensional stratified space, any codimension $k$ geometric cochain $\theta$ in $X$ intersects each stratum of $X$ in a set of codimension $\geqslant k$. Geometric $n-k$ dimensional cycles which satisfy only this dimensionality condition are said to have perversity $\overline{0}$. This is a partial relaxation of the $\pi$-fibre condition. In Goresky and MacPherson [8] it is shown that if $X$ is a P.L. oriented normal pseudo-manifold then the homology of the complex of geometric chains with perversity $\overline{0}$ is naturally isomorphic to the cohomology of $X$. Further relaxations of the $\pi$-fibre condition are considered in [8] which result in new "homology" groups $I H_{*}^{\bar{p}}(X)$.

5. Transversality and the pullback. In this section we show that for any controlled stratified map $f: X_{1} \rightarrow X_{2}$ the induced map $f^{*}: H^{k}\left(X_{2}\right) \rightarrow H^{k}\left(X_{1}\right)$ is given by choosing a representative geometric cocycle in $X_{2}$ which is transverse to $f$, (whose existence is guaranteed by 5.2) and taking the pre-image of that cocycle.

5.1. Definition. Suppose $X_{1}$ and $X_{2}$ are Whitney objects (in some manifold). A map $f: X_{1} \rightarrow X_{2}$ will be called stratified if, for each stratum $R$ of $X_{1}$ there is a stratum $S$ of $X_{2}$ such that $f(R) \subset S$ and $f \mid R: R \rightarrow S$ is smooth.

Suppose in addition that a system of control data is specified on $X_{1}$ and $X_{2}$. Then a stratified map $f: X_{1} \rightarrow X_{2}$ is controlled if for each stratum $S$ of $X_{2}$ and $R$ of $X_{1}$ there is an $\varepsilon>0$ such that whenever $p \in T_{R}(\varepsilon)$ and $f(p) \in T_{S}(\varepsilon)$ we have $\pi_{S} f(p)=f \pi_{R}(p)$.

If $f: X_{1} \rightarrow X_{2}$ is a stratified map and if $Y \subset X_{2}$ is a Whitney substratified object we will say $f$ is transverse to $Y$ provided for every stratum $A$ of $Y$ and every stratum $S_{1}$ of $X_{1}$, either $f\left(S_{1}\right) \cap A=\varnothing$ or $f \mid S_{1}: S_{1} \rightarrow S_{2}$ is transverse to $A$ (here $S_{2}$ denotes the stratum of $X_{2}$ which contains $A$ ). 
5.2. Proposition. Suppose $f: X_{1} \rightarrow X_{2}$ is a controlled stratified map and let $Y$ be a codimension $k$ geometric cocycle in $X_{2}$ such that $f$ is transverse to $|Y|$. Then $f^{-1}(|Y|)$ is $a \pi$-fibre subset of $X_{1}$ which admits a canonical Whitney stratification. The coorientation of $Y$ pulls back to a coorientation on $f^{-1}(|Y|)$ which then becomes a geometric cocycle $f^{-1}(Y)$ and it represents the cohomology class $f^{*}([Y])$ in $H^{k}\left(X_{1}\right)$.

Proof. The strata of $f^{-1}(|Y|)$ (with intrinsic codimension $p$ ) are the components of the manifolds $S_{1} \cap f^{-1}(A)$ where $S_{1}$ is any stratum of $X_{1}$ and $A$ is any stratum of $|Y|$ (with intrinsic codimension $p$ ). The Whitney conditions can be easily verified using the fact that $f^{-1}(|Y|)$ satisfies the $\pi$-fibre condition. For each codimension $k$ costratum $D$ of $Y, f^{-1}(D)$ is a codimension $k$ costratum of $f^{-1}(|Y|)$ whose oriented normal bundle, Thom class, and coboundary is the pullback of the oriented normal bundle, Thom class and coboundary of $D$, by the naturality of the Thom isomorphism theorem. Thus $\left[f^{-1}(Y)\right]=f^{*}[Y]$.

5.3. Transversality Lemma. Suppose $X_{1}$ and $X_{2}$ are Whitney stratified subsets of the manifolds $M_{1}$ and $M_{2}$ (respectively). Fix a system of control data on $X_{1}$ and $X_{2}$ and let $f: X_{1} \rightarrow X_{2}$ be a stratified map. Suppose $Y \subset X_{2}$ is a geometric cocycle. Suppose either (a) $f$ is controlled or (b) $f$ is the restriction of a smooth map $\tilde{f}$ : $M_{1} \rightarrow M_{2}$. Then $Y$ is cobordant to a cocycle $Y^{\prime} \subset X_{2}$ such that $f$ is transverse to $Y^{\prime}$.

Proof. Assume by induction on $k$ that $Y$ is cobordant to a cocycle $Y_{k}$ such that $f$ is transverse to $Y_{k} \cap\left(X_{2}\right)_{k}$, where $\left(X_{2}\right)_{k}$ denotes the $k$ skeleton of $X_{2}$. We will find a controlled vector field $\eta$ on $X_{2}$ with (controlled) flow $F_{t}: X_{2} \rightarrow X_{2}$ such that $Y_{k+1}=F_{1}\left(Y_{k}\right)$ satisfies the induction hypothesis. Under either assumption (a) or (b) above, there is a neighborhood $U$ of $\left(X_{2}\right)_{k}$ such that $f$ is transverse to $Y_{k} \cap U$. Let $S=\left(X_{2}\right)_{k+1}-\left(X_{2}\right)_{k}$. By Sards's theorem [6] there is a vector field $\eta_{S}$ on $S$ with time 1 flow $F_{S}: S \rightarrow S$ such that $\eta_{S}$ vanishes near $\left(X_{2}\right)_{k}$ and such that $f$ is transverse to $F_{S}\left(Y_{k}\right)$. Take $\eta$ to be any controlled lift [5], [13] of $\eta_{S}$.

6. Products. In this section we fix a Whitney object $X$ with a system of control data and show (6.2) that the cup product in $H^{*}(X)$ is given by transversal intersection of geometric cochains, while the cap product (6.3) is given by transversal intersection of geometric cochains with geometric chains.

6.1. Definition. Let $M_{1}$ and $M_{2}$ be transversally intersecting submanifolds of a manifold $N$ and suppose their normal bundles $\nu_{1}$ and $\nu_{2}$ are oriented. Let $\Delta$ : $M_{1} \cap M_{2} \rightarrow M_{1} \times M_{2}$ be the map $\Delta(x)=(x, x)$ so the normal bundle of $M_{1} \cap$ $M_{2}$ is $\Delta^{*}\left(\nu_{1} \oplus \nu_{2}\right)$. Define the product coorientation of $M_{1} \cap M_{2}$ to be the pullback of the direct sum orientation of $\nu_{1} \oplus \nu_{2}$.

Similarly if $\nu_{1}$ is oriented and if the tangent bundle $\tau_{2}$ of $M_{2}$ is also oriented define the product orientation of the tangent bundle $\tau_{12}$ of $M_{1} \cap M_{2}$ so that $\tau_{12} \oplus \nu_{1} \cong \tau_{2}$ is an isomorphism of oriented vector bundles over $M_{1} \cap M_{2}$.

6.2. Proposition. Suppose $Y_{1}$ and $Y_{2}$ are geometric cocycles in a Whitney object $X$. Then $Y_{2}$ is cobordant to a cocycle $Y_{2}^{\prime}$ which is transverse to $Y_{1}$. In this case $Y_{1} \cap Y_{2}^{\prime}$ is a geometric cocycle under the product coorientation and $\left[Y_{1} \cap Y_{2}^{\prime}\right]=\left[Y_{1}\right]$ $\cup\left[Y_{2}^{\prime}\right]$. 
Proof. The inclusion $\left|Y_{1}\right| \rightarrow X$ satisfies the hypotheses 5.3 on $f$, so $Y_{2}$ is cobordant to a cocycle $Y_{2}^{\prime}$ which is transverse to $Y_{1}$. This means the diagonal $\Delta$ : $X \rightarrow X \times X$ is transverse to the cocycle $Y_{1} \times Y_{2}^{\prime}$ so the pullback $\Delta^{-1}\left(Y_{1} \times Y_{2}^{\prime}\right)=$ $Y_{1} \cap Y_{2}^{\prime}$ represents the cohomology class $\Delta^{*}\left(\left[Y_{1}\right] \times\left[Y_{2}\right]\right)=\left[Y_{1}\right] \cup\left[Y_{2}\right]$.

6.3. Proposition. Suppose $Y$ is a codimension $k$ geometric cocycle in $X$, and $Z$ is a $p$ dimensional geometric cycle. Then $Y$ is cobordant to a cocycle $Y^{\prime}$ which is transverse to $Z$. Using the product orientation on the $p-k$ dimensional strata of $Y^{\prime} \cap Z$ (which all have the form (codimension $p$ costratum of $\left.Y^{\prime}\right) \cap$ ( $k$ dimensional stratum of $Z)$ ), $Y^{\prime} \cap Z$ becomes a geometric cycle and it represents the cap product $[Y] \cap[Z] \in H_{p-k}(X)$.

Proof. It is straightforward to check the boundary multiplicites on the strata of $\left|Y^{\prime} \cap Z\right|_{p-k-1}$ are all 0 . Let $i:|Z| \rightarrow X$ be the controlled inclusion, so $i^{*}\left(Y^{\prime}\right)$ is a geometric cocycle in $Z$ representing the class $i^{*}[Y]$. It is sufficient to show that the geometric cycle $\left|i^{*}\left(Y^{\prime}\right)\right|$ with its product orientation represents the homology class $\left[i^{*}\left(Y^{\prime}\right)\right] \cap[Z]$ in $Z$, because then the projection formula gives

$$
\left[Y^{\prime} \cap Z\right]=i_{*}\left(\left[i^{*}\left(Y^{\prime}\right)\right] \cap[Z]\right)=i_{*}\left(i^{*}\left[Y^{\prime}\right] \cap[Z]\right)=[Y] \cap[Z] \text {. }
$$

Let $A$ be a codimension $k$ costratum of $i^{*}\left(Y^{\prime}\right)$ with normal bundle $\pi: E \rightarrow A$. We must show the product orientation on $A \cap\left(|Z|_{p}-|Z|_{p-1}\right)$ corresponds to the cap product of $\left[i^{*}\left(Y^{\prime}\right)\right]$ with the fundamental class $[Z]$. This is a local question so we may assume that $\left[i^{*}\left(Y^{\prime}\right)\right]$ is a multiple of the Thom class $\mu_{A} \in H^{k}(E, E-A)$. After this reduction, the necessary lemma appears in Milnor [15, §10.7].

7. Proof of Theorem 4.7 for compact $X$. In this section $X$ denotes a compact $n$-dimensional Whitney object with a fixed system of control data, which is contained in an $m$-dimensional manifold $M$ (as in \$4). We may assume $M$ is oriented. We will find a "nice" neighborhood $U$ of $X$ such that $H^{k}(X) \cong H^{k}(U) \cong$ $H_{m-k}(U, \partial U)$. Geometric cycles in $(U, \partial U)$ correspond (by transversally intersecting them with $X$ ) to geometric cocycles in $X$, thus reducing Theorem 4.7 to the (relative version of) Theorem 3.4 .

7.1. Definition. Let $U(\varepsilon)=\cup\left\{T_{A}(\varepsilon) \mid A\right.$ is a stratum of $\left.X\right\}$. For sufficiently small $\varepsilon$ (which we now fix), $U(\varepsilon)$ is an $m$-manifold with corners: its boundary $\partial U(\varepsilon)$ has a "corner" $S_{A_{1}}(\varepsilon) \cap S_{A_{2}}(\varepsilon) \cap \cdots \cap S_{A_{k}}(\varepsilon)$ for each string of incident strata $A_{1}<A_{2}<\cdots<A_{k}$ (see $\S 2$ ). We now construct an important deformation retraction $f: U(\varepsilon) \rightarrow X$. Choose a family of lines subordinate to the control data on $X$. This consists of a stratum preserving retraction, $r_{A}: T_{A}(2 \varepsilon)-A \rightarrow S_{A}(2 \varepsilon)$ for each stratum $A$ of $X$, such that whenever $A<B$ the following conditions hold: $r_{A} \mid B$ is smooth, $r_{A} r_{B}=r_{B} r_{A}, \rho_{A} r_{B}=\rho_{A}, \rho_{B} r_{A}=\rho_{B}, \pi_{A} r_{A}=\pi_{A}$, and $\pi_{A} r_{B}=\pi_{A}$. Families of lines are constructed in Goresky [7]. Associated to a family of lines are stratum preserving homeomorphisms $h_{A}: T_{A}(2 \varepsilon)-A \rightarrow S_{A}(2 \varepsilon) \times(0,2 \varepsilon)$ defined by $h_{A}(p)$ $=\left(r_{A}(p), \rho_{A}(p)\right)$ which collar each of the corners of $U(\varepsilon)$. Each $h_{A}$ extends to a homeomorphism (also denoted $h_{A}$ ) between $T_{A}(2 \varepsilon)$ and the mapping cylinder (with height $2 \varepsilon$ ) of the map $\pi_{A}: S_{A}(2 \varepsilon) \rightarrow A$. 
Fix a smooth nondecreasing function $q$ such that $q(t)=0$ for $t \leqslant \varepsilon$ and $q(t)=t$ for $t \geqslant 2 \varepsilon$. For each stratum $A$ define

$$
H_{A}(p)=\left\{\begin{array}{l}
p \quad \text { if } p \notin T_{A}\left(2_{\varepsilon}\right), \\
h_{A}^{-1}\left(r_{A}(p), q\left(\rho_{A}(p)\right)\right) \quad \text { if } p \in T_{A}(2 \varepsilon) .
\end{array}\right.
$$

$H_{A}$ is continuous, homotopic to the identity, and if $p \in T_{A}(\varepsilon)$ then $H_{A}(p)=\pi_{A}(p)$. Define $\tilde{f}: U(2 \varepsilon) \rightarrow U(2 \varepsilon)$ to be the composition $H_{A_{1}} \circ H_{A_{2}} \circ \cdots \circ H_{A_{n}}$ where the $A_{i}$ run over all the strata of $X$ (in any order). Define $f$ to be the restriction of $\tilde{f}$ to $U(\varepsilon)$.

7.2. Properties of $f$. (a) $f$ is a deformation retraction $U(\varepsilon) \rightarrow X$ since each $H_{A}$ takes $T_{A}(\varepsilon)$ to $A$.

(b) If $i: X \rightarrow U(\varepsilon)$ denotes the inclusion, then $g=i \circ(f \mid X): X \rightarrow U(\varepsilon)$ is controlled, i.e. for any $x \in T_{A}(\varepsilon) \cap X$ we have $g(x)=g\left(\pi_{A}(x)\right)$.

(c) For any geometric cocycle $Y$ in $X$, the set $f^{-1}(|Y|)$ is the support of a ( $\pi$-fibre) geometric cocycle in $U(\varepsilon)$, whose cohomology class is $f^{*}[Y] . f^{-1}(|Y|)$ respects the collaring of each of the corners of $U(\varepsilon)$.

7.3. To show $W H^{k}(X) \rightarrow H^{k}(X)$ is surjective, fix $\alpha \in H^{k}(X)$. By 3.5 there is a relative geometric $m-k$ cycle $Y$ in $(U(\varepsilon), \partial U(\varepsilon))$ which represents the class $f^{*}(\alpha) \cap[U(\varepsilon), \partial U(\varepsilon)]$. Fix the coorientation on $|Y|$ which makes it into a geometric cocycle $\tilde{Y}$, representing the class $f^{*}(\alpha) \in H^{k}(U(\varepsilon))$. By Lemma 5.3 we may assume $g: X \rightarrow U(\varepsilon)$ is transverse to $\tilde{Y}$. Since $g$ is controlled, it follows that $g^{*}(\tilde{Y})$ is a geometric cocycle which represents the class $g^{*}[\tilde{Y}]=g^{*} f^{*}(\alpha)=\alpha$.

To show $W H^{k}(X) \rightarrow H^{k}(X)$ is injective, suppose $Y_{1}$ and $Y_{2}$ are geometric cocycles in $W H^{k}(X)$ which represent the same cohomology class in $X$. Then $f^{*}\left(Y_{i}\right)$ is a geometric cocycle in $U(\varepsilon)$ representing $f^{*}\left[Y_{i}\right]$. Using the product orientation, $\left|f^{*}\left(Y_{i}\right)\right|$ become homologous relative geometric cycles in $H_{m-k}(U(\varepsilon), \partial U(\varepsilon))$. By 3.5 there is a relative cobordism $W$ between them, and we may assume $g: X \rightarrow U(\varepsilon)$ is transverse to $W$. Consequently, coorienting $g^{*}(W)$ as above gives a $(\pi$-fibre) cobordism between $Y_{1}$ and $Y_{2}$.

\section{Applications.}

8.1. Failure of Poincare Duality. Suppose an $n$-dimensional Whitney object $X$ is itself a compact oriented geometric cycle. The cap product with the fundamental class $[X]$ defines the Poincaré Duality map $H^{k}(X) \rightarrow H_{n-k}(X)$. Theorems 4.7 and 6.3 then give (see McCrory [11] and Sullivan [17]):

(A) A homology class is in the image of the Poincare Duality map iff it has a $\pi$-fibre geometric cycle representative.

(B) A cohomology class is in the kernel of the Poincare Duality map if it can be represented by a geometric cocycle which is the boundary of a geometric chain but is not the boundary of a $\pi$-fibre geometric chain.

For example, cycle $A$ illustrated in the pinched torus below is not in the image of Poincare Duality while cocycle $B$ is in the kernel of Poincaré Duality. 


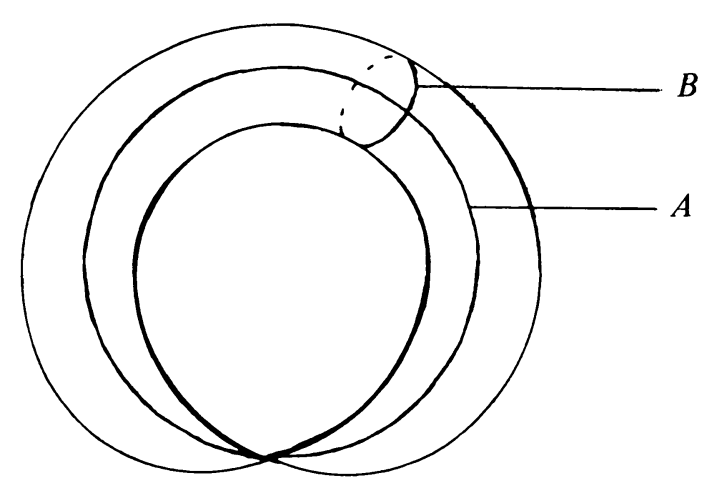

The pinched torus

8.2. Relative cap product. Let $X$ be a stratified space and let $A$ be a closed union of strata. Suppose $W H_{k}(X) \rightarrow H_{k}(X)$ is surjective (e.g. if $X$ is subanalytic or if $X$ is a simplicial complex). If $B$ is also a closed union of strata then $H^{q}(X, B)$ is represented by geometric cocycles in $X-B$ (4.9). Thus transverse intersection determines a cap product

$$
H^{q}(X, B) \times H_{p}(X, A) \rightarrow H_{p-q}(X-B, A-B) .
$$

This cap product appears in Whitehead [24].

8.3. Stratification of $K(\mathbf{Z}, n)$. According to the Dold-Thom theorem [2], a finite approximation to the Eilenberg-Mac Lane space $K(Z, n)($ for $n>2)$ is given by the quotient space of the $p$-fold Cartesian product $M_{p}=S^{n} \times S^{n} \times \cdots \times S^{n}$ (for large $p$ ) by the permutation group $\Gamma_{p}$. Associated to this action is a canonical stratification of the projection $\pi: M_{p} \rightarrow M_{p} / \Gamma_{p}$ which is locally equivalent to the projection $T_{x} M_{p} \rightarrow T_{x} M_{p} / S(x)$ where $S(x)$ is the stabilizer of $x \in M_{p}$ acting on $T_{x} M_{p}$ by the differential of the action of $\Gamma_{p}$. In fact, for each partition $I$ of the set $\{1,2, \ldots, p\}$ into nonempty disjoint subsets $I_{1} \cup I_{2} \cup \cdots \cup I_{k}$ there is a stratum of $M_{p}$ which consists of those points $x=\left(x_{1} \ldots x_{p}\right)$ such that for each grouping $I_{q}=\left\{i_{1}, i_{2}, \ldots, i_{l}\right\}$ we have $x_{i_{1}}=x_{i_{2}}=\cdots=x_{i_{i}}$. The dimension of this stratum is $n k . \Gamma_{p}$ identifies the strata corresponding to partitions $I$ and $J$ precisely when the formulas $\left|I_{1}\right|+\left|I_{2}\right|+\cdots+\left|I_{k}\right|=p$ and $\left|J_{1}\right|+\left|J_{2}\right|+\cdots+\left|J_{k}\right|=p$ are the same.

Let $0 \in S^{n}$ denote the south pole, or basepoint.

The subset $M_{p} \subset M_{p+1}$ given by $x_{p+1}=0$ is not a union of strata, however it is transverse to the stratification of $M_{p+1}$ which therefore pulls back to a stratification of $M_{p}$. This new stratification of $M_{p}$ refines the stratification defined previously and $\Gamma_{p}$ respects this refinement, so $M_{p} / \Gamma_{p}$ becomes a substratified object in $M_{p+1} / \Gamma_{p+1}$. It is also clear that $M_{p} / \Gamma_{p}$ is dimensionally $\pi$-fibre in $M_{p+1} / \Gamma_{p+1}$ since it intersects each stratum of $M_{p+1} / \Gamma_{p+1}$ in the same codimension, $n$. Although it is not $\pi$-fibre for any system of control data it has perversity $\overline{0}$ and represents a cohomology class since $M_{p+1} / \Gamma_{p+1}$ is normal. See $\S 4.10$.

THEOREM. For large $p,\left[M_{p} / \Gamma_{p}\right]$ in $M_{p+1} / \Gamma_{p+1}$ is the pullback of the "universal" cohomology class $U \in H^{n}(K(\mathbf{Z}, n))$. Similarly $\left[M_{p-1} / \Gamma_{p-1}\right]$ represents $U^{2}$. 
Proof. $\pi_{n}\left(M_{p+1} / \Gamma_{p+1}\right)$ is generated by the embedding of $S^{n}$ given by $x \rightarrow$ $[x, a, a, \ldots, a]$ where $0 \neq a \in S^{n}$. However $M_{p} / \Gamma_{p}$ intersects this in exactly one point $[0, a, \ldots, a]$ with multiplicity one, so it represents the dual cohomology class.

Remarks. The inclusion $M_{p} \subset M_{p+1}$ does not respect orbit type so $M_{p} / \Gamma_{p}$ is not a $\pi$-fibre subset of $M_{p+1} / \Gamma_{p+1}$. It may happen that if $X<Y$ are strata of $M_{p+1} / \Gamma_{p+1}$ and if $S \subset X$ is a stratum of $M_{p} / \Gamma_{p}$ then there are several strata $T_{1}, T_{2} \subset Y$ such that $S<T_{i}$. Further study of these "extra singularities" should lead to a canonical list of singularities which can be used to represent all homology classes, as in Sullivan [18]. The case $n=2$ is the well known example $\mathbf{C} P^{p} \subset$ $\mathbf{C} P^{p+1}$ which is free of singularities.

8.4. Controlled differential forms. For a Whitney object $X$ with a system of control data, define a controlled differential $p$-form $\omega$ to be a collection of smooth $p$-forms $\omega_{A} \in \Omega^{p}(A)$ for each stratum $A$ of $X$ such that whenever $A<B$ and whenever $x \in T_{A}(\varepsilon) \cap B, \omega_{B}(x)=\left(\pi_{A}^{*} \omega_{A}\right)(x)$. There is an obvious analogy between $\pi$-fibre cochains and controlled differential forms. In [23], Verona proves that the homology of the complex of controlled differential forms is precisely the (real) cohomology of $X$. Using [22] and the method of $\$ 7$ it is easy to see that any controlled differential $p$-form $\omega$ can be integrated over any geometric $p$-chain $Y \subset X$, that $\int_{\partial Y} \omega=\int_{Y} d \omega$ and if $d \omega=\partial Y=0$ then $\int_{Y} \omega=[\omega] \cap[Y] \in \mathbf{R}$.

8.5. Gysin homomorphisms. Suppose $Y \subset X$ is a geometric cocycle of codimension $k$. Define Gysin homomorphisms $f_{!}: H^{a}(Y) \rightarrow H^{a+k}(X)$ and $f^{!}: W H_{b}(X) \rightarrow$ $W H_{b-k}(Y)$ as follows: any codimension a geometric cocycle $\xi$ in $Y$ is also $\pi$-fibre in $X$. The direct sum of the coorientation of $\xi$ in $Y$ with the coorientation of $Y$ in $X$ gives a coorientation of $\xi$ in $X$, and the same applies to cobordisms. Similarly, if $\eta$ is a $b$ dimensional geometric cycle in $X$ we can make $Y$ transverse to $\eta$. The product orientation on $Y \cap|\eta|$ defines a geometric $b-k$ cycle in $Y$.

These homomorphisms satisfy the usual relations, for example $f_{1} f^{*}: H^{p}(X) \rightarrow$ $H^{p+k}(X)$ is the cup product with $[Y] . f^{*} f_{!}: H^{a}(Y) \rightarrow H^{a+k}(Y)$ is the cup product with $f^{*}[Y]$ which (if $Y$ is normally nonsingular in $X(8.6)$ ) is the Euler class of the normal bundle of $Y$.

Gysin homomorphisms also exist for any fibre bundle $f: Y \rightarrow X$ when the fibre is a compact oriented geometric cycle of dimension $k . f^{!}: W H_{a}(X) \rightarrow W H_{a+k}(Y)$ assigns to any geometric $a$-cycle in $X$ its (oriented) pre-image in $Y . f_{!}: H^{a}(Y) \rightarrow$ $H^{a-k}(X)$ is harder to define (intuitively, one takes the image of geometric cocycles in $Y$ ). Suppose $Y$ is compact. Choose an embedding $i: Y \subset \mathbf{R}^{N}$. Then $(f \times i)$ : $Y \rightarrow X \times \mathbf{R}^{N}$ is a codimension $N-k \pi$-fibre inclusion (for some system of control data on $X \times \mathbf{R}^{N}$ ) with normal orientation. Identify $\mathbf{R}^{N}$ with the Euclidean ball $B^{N}$ whose boundary is $S^{N-1}$, and use the Kunneth theorem to obtain homomorphisms

$$
H^{a}(Y) \rightarrow H^{a+N-k}\left(X \times B^{N}, X \times S^{N-1}\right) \cong H^{a-k}(X) .
$$

8.6. Normally nonsingular maps. The results in this section are due to Bob MacPherson (1974). We shall show that the cobordism theory of geometric cochains with "pure" codimension $m+k$ in the space $X \times \mathbf{R}^{m}$ identifies with the smooth oriented (Thom-) cobordism group $\Omega^{k}(X)$ for large $m$. The P.L. version of this theorem may be found in $[\mathbf{1}, \S I I]$. 
Definition. Let $X$ be a Whitney object and suppose $f: Y \rightarrow X$ is a stratified map. $f$ is a codimension $k$ normally nonsingular inclusion if it embeds $Y$ as a codimension $k$ geometric cocycle in $X$ (with respect to some system of control data) such that $Y$ has only costrata of codimension $k$ (and we say $Y$ has pure codimension $k$ ). $f$ is a normally nonsingular map of codimension $k$ if it can be factored as a normally nonsingular inclusion of codimension $k+m, Y \rightarrow X \times \mathbf{R}^{m}$, followed by projection to the first factor. In this case, $Y$ has a normal bundle $E$ which embeds as an open tubular neighborhood of $Y$ in $X \times \mathbf{R}^{m}$. The Gysin maps of 8.7 can be constructed classically by applying the Thom isomorphism and Kunneth theorem to the factorization $Y \subset E \subset X \times \mathbf{R}^{m} \rightarrow X$. Any fibration with smooth compact oriented fibre is normally nonsingular.

Two codimension $k$ normally nonsingular maps $Y_{0} \rightarrow X$ and $Y_{1} \rightarrow X$ are cobordant if there is a purely codimension $k$ cobordism $V \subset X \times \mathbf{R}^{N} \times[0,1]$ whose coboundary is $Y_{1}-Y_{0}$ (here we have identified $\mathbf{R}^{m}$ with a coordinate plane in $\mathbf{R}^{N}$ ).

THEOREM. The cobordism classes of codimension $k$ normally nonsingular maps to $X$, are in one-to-one correspondence with elements of the (smooth) cobordism group $\Omega^{k}(X)$.

The proof is essentially a duplication of $\S 7$. Embed $X$ as a Whitney object in $S^{N}$ with "regular" neighborhood $U$ and retraction $f: U \rightarrow X$. Since $\Omega^{k}(X) \simeq$ $\Omega_{N-k}\left(S^{N}, S^{N}-X\right)$, any cobordism element can be represented by a map $g$ : $(M, \partial M) \rightarrow\left(S^{N}, S^{N}-X\right)$ where $M$ is an $N-k$ dimensional manifold which we may embed in some $\mathbf{R}^{m}$. This gives an embedding $\tilde{g}:(M, \partial M) \rightarrow\left(S^{N} \times \mathbf{R}^{m},\left(S^{N}\right.\right.$ $-X) \times \mathbf{R}^{m}$ ) which can be made transverse and $\pi$-fibre with respect to $X \times \mathbf{R}^{m}$. Then the projection of $Y=\tilde{g}(M) \cap\left(X \times \mathbf{R}^{m}\right)$ to $X$ is a normally nonsingular map of codimension $k$.

Similarly if $Y \subset X \times \mathbf{R}^{m}$ is a codimension $k+m$ normally nonsingular inclusion then $\tilde{f}^{-1}(Y)$ is a submanifold of $U \times \mathbf{R}^{m}$ with boundary in $\partial U \times \mathbf{R}^{m}$ so it determines an element of $\Omega_{N-k}\left(U \times \mathbf{R}^{m}, \partial U \times \mathbf{R}^{m}\right)=\Omega^{k}(X)$. (Here, $\tilde{f}=f \times$ id: $U \times \mathbf{R}^{m} \rightarrow X \times \mathbf{R}^{m}$.)

REMARK. For any bordism theory with singularities [1] the corresponding cobordism theory can be given a $\pi$-fibre geometric cocycle description using this same procedure. This program is carried out with P.L. techniques in [1].

A complementary treatment of normally nonsingular maps can be found in Fulton and MacPherson [4].

9. $\pi$-fibre Morse theory. The "Morse functions" considered in this section are assumed to increase in the directions normal to each stratum. We show how $X$ can be built up by a combination of stratum preserving deformations (where there are no critical points) and by adding certain $\pi$-fibre subsets (when crossing a critical point). This decomposition gives rise to the usual Morse inequalities relating the Betti numbers of $X$ to the indices of the critical points. More general Morse functions are considered by Goresky and MacPherson in Stratified Morse Theory (preprint, Univ. of B.C., 1980). 
9.1. $\pi$-fibre Morse functions. Fix a Whitney object $X$ with a system of control data. Fix $\varepsilon>0$. For each stratum $A$ of $X$, define

$$
A^{\circ}=A-\cup T_{B}(2 \varepsilon), \quad T_{A}^{\circ}=T_{A}(\varepsilon)-\cup T_{B}(2 \varepsilon)
$$

where the union is taken over all strata $B<A$.

A $\pi$-fibre Morse function $f: X \rightarrow \mathbf{R}$ is defined to be a continuous function such that for each stratum $A$ of $X, f \mid A$ is a smooth Morse function whose critical points lie in $A^{\circ}$, and such that for any $x \in T_{A}^{\circ}$, we have

$$
f(x)=f\left(\pi_{A}(x)\right)+\rho_{A}(x) .
$$

An easy induction shows that such functions exist and have smooth extensions to the ambient manifold which contains $X$. For the remainder of this chapter we fix such a function $f$.

For each $c \in \mathbf{R}$ let $X_{c}=\{x \in X \mid f(x) \leqslant c\}$. We shall say $c$ is a critical value of $f$ if it is a critical value of $f \mid A$ for any stratum $A$. For example, each 0-dimensional stratum is a critical point (a local minimum, in fact).

9.2. THEOREM. Suppose $[a, b]$ contains no critical values of $f$ and suppose $f^{-1}[a, b]$ is compact. Then there is a stratum preserving deformation retraction $X_{b} \rightarrow X_{a}$.

PRoof. For some $\delta>0$ the open set $U=f^{-1}(a-\delta, b+\delta)$ contains no critical points, so $f \mid U$ is a submersion on each stratum. By Mather [13] there is a (new) system of control data on $U$ such that $f$ becomes controlled, and there is a controlled vector field $V$ on $U$ such that $f_{*}(V)=-d / d t$. The (controlled) flow of $V$ is the desired deformation.

CoRollary. Suppose $X$ is compact and $g: X \rightarrow \mathbf{R}$ is the restriction of some smooth function defined on a neighborhood of $X$. Then $g$ has at least cat $(X)$ critical points. Here $\operatorname{cat}(X)$ denotes the Lyusternik-Schnirelman category of $X$, and $\operatorname{cat}(X) \geqslant$ cuplength $(X)+1$.

9.3. Suppose $y$ is an isolated critical point of $f \mid A$ for some stratum $A$. For each $\delta>0$ let $N_{\delta}$ denote the normal slice through $A$ (at the point $y$ ) of radius $\delta$ :

$$
N_{\delta}=\pi_{A}^{-1}(y) \cap X \cap T_{A}(\delta) .
$$

It inherits a stratification and control data from $X$.

Let $c=f(y)$ be the critical value and suppose that for some $\delta>0$,

$$
f^{-1}[c-\delta, c+\delta]
$$

is compact and contains no critical points other than $y$. Let $\lambda$ be the index of $f$ at $y$, and let $e_{\lambda}$ be a $\lambda$-cell.

THEOREM. For $\delta$ sufficiently small, there is a stratum preserving deformation retraction $X_{c+\delta} \rightarrow X_{c-\delta} \cup\left(e_{\lambda} \times N_{\delta}\right)$ where $e_{\lambda} \times N_{\delta}$ is embedded in a stratum preserving manner (with $e_{\lambda} \times\{y\} \subset A$ ) and is attached to $X_{c-\delta}$ along $\left(\partial e_{\lambda}\right) \times N_{\delta}$.

Proof. Choose a coordinate neighborhood $B \subset A^{\circ}$ of $y$ which contains no other critical points, and for which there is a controlled local trivialization $\psi: B \times N \rightarrow$ $\pi_{A}^{-1}(B)$. For $\delta$ sufficiently small we may assume (as in Milnor [6]) that there is a 
local coordinate system on $B$ such that $B=\left\{x \mid \sum x_{i}^{2} \leqslant 2 \delta\right\}$ and $f \mid B=c+\Sigma \pm x_{i}^{2}$. In particular, Milnor finds a cell $e_{\lambda} \subset B$ which lies in a coordinate plane, such that $e_{\lambda} \cap A_{c-\delta}=\partial e_{\lambda}$. The map $\psi \mid\left(e_{\lambda} \times N\right)$ embeds $e_{\lambda} \times N_{\delta}$ into $X$ but it must be "stretched" near the edges so as to take $\left(\partial e_{\lambda}\right) \times N_{\delta}$ into $X_{c-\delta}$.

Choose a smooth function $\eta: B \rightarrow[0,1]$ such that $\eta(x)=0$ if $\Sigma x_{i}^{2}<\delta / 4$, and $\eta(x)=1$ if $\sum x_{i}^{2} \geqslant \delta / 2$. Then $g(x)=f\left(\pi_{A}(x)\right): \pi_{A}^{-1}(B) \rightarrow \mathbf{R}$ is a controlled submersion (except when $\pi_{A}(x)=y$ ) so there is a controlled vector field $V$ such that $g_{*}(v)=-d / d t$. If $H_{t}$ denotes the controlled flow of $V$, we have $H_{0}(x)=x$, and $f\left(H_{\rho(x)}(x)\right)=f\left(\pi_{A}(x)\right)$ for all $x \in \pi_{A}^{-1}(B)$. The desired embedding $\theta: e_{\lambda} \times N \rightarrow X$ is given by

$$
\theta(x, z)=H_{\eta(x) \rho(z)}(\psi(x, z))
$$

because if $x \in \partial\left(e_{\lambda}\right)$ then $f(\psi(x, z))=f(x)=c-\delta$.

Similarly we obtain a stratified embedding $H \times N_{\delta} \rightarrow X$ (where $H$ is the handle associated to $e_{\lambda}$ as in Milnor [16]), such that $e_{\lambda} \times N_{\delta}$ is a deformation retract of $H \times N_{\delta}$. Finally a stratum preserving retraction $X_{c+\delta} \rightarrow X_{c-\delta} \cup\left(H \times N_{\delta}\right)$ can be constructed as in 9.2 using the controlled flow of $\nabla F$ for some small modification $F$ of the function $f$ (as in Milnor).

9.4. If we drop the restriction that all deformation retracts be stratum preserving, then $e_{\lambda}$ becomes a deformation retract of $e_{\lambda} \times N_{\delta}$. Thus we have

COROLlary. $X$ has the homotopy type of a $\mathrm{CW}$ complex with a cell of dimension $\lambda$ for each critical point of index $\lambda$. If $C_{\lambda}$ denotes the number of such critical points then $\operatorname{rank}\left(H_{\lambda}(X)\right) \leqslant C_{\lambda}$ and $\chi(X)=\Sigma(-1)^{\lambda} C_{\lambda}$.

9.5. The "dual decomposition" of $X$ is obtained by examining the sets $X^{c}=\{x$ $\in X \mid f(x) \geqslant c\}$. Suppose, as in 9.3 that $y \in A$ is a critical point of index $\lambda$, critical value $c$, and that $N$ is a normal slice. Then using the techniques of 9.3 it is easy to see that $X^{c-\delta}$ deformation retracts to $X^{c+\delta} \cup\left(e_{\alpha-\lambda} \times N\right)$ where $\alpha=\operatorname{dim}(A)$ and the "co-cell" $e_{\alpha-\lambda} \times N$ is attached to $X^{c+\delta}$ along $\left(\partial e_{\alpha-\lambda}\right) \times N \cup e_{\alpha-\lambda} \times(\partial N)$.

9.6. Remark. One would like to duplicate the Thom-Smale theory of stable and unstable manifolds, for the case of the gradient of a $\pi$-fibre Morse function. For example one could fix a conical Riemannian metric on each stratum $A$ (see Appendix). Then $-\nabla\left(f \circ \pi_{A}\right)$ and $-\nabla \rho_{A}$ become commuting vector fields so their sum, $-\nabla f$, defines a (noninvertible) "flow" $F_{t}$ which is far from being controlled. It collapses a neighborhood $T_{A}(\varepsilon)$ to $A$ in finite time. Nevertheless we can define, for each nondegenerate critical point $y$, a "stable variety" $S_{y}=\left\{x \mid \lim _{t \rightarrow \infty} F_{t}(x)=y\right\}$. $S_{y}$ is a $\pi$-fibre subset of $X$ with codimension $\lambda=\operatorname{index}(y)$.

Conjecture. Every Whitney stratified set $X$ admits a Riemannian metric and $\pi$-fibre Morse function such that for each $k$, the set

$$
W^{k}=\cup\left\{S_{y} \mid y \text { is a critical point of index } \geqslant k\right\}
$$

is a $\pi$-fibre Whitney stratified subset, and every codimension $k$ geometric cocycle can be deformed into it.

Appendix: conical singularities. In this Appendix we show that any Whitney stratified subset of a smooth manifold can be deformed to a Whitney stratified set which has conical singularities, and the whole deformation can be made to satisfy 
the Whitney conditions. Furthermore, the deformed stratified set (which has conical singularities) can be decomposed into a union of cells such that the closure of each cell is a Whitney stratified set.

A.1. Mapping cylinders of stratified submersions.

Definition. Suppose $f: M_{1} \rightarrow M_{2}$ is a smooth fibration and suppose $W_{i} \subset M_{i}$ are Whitney objects. We say $f$ is a stratified submersion if $f\left(W_{1}\right)=W_{2}$ and if for each stratum $A_{1}$ of $W_{1}, f \mid A_{1}$ is a submersion onto a stratum of $W_{2}$. The stratified submersion $f$ is said to satisfy condition $\mathrm{D}$ if the following holds for each pair $A_{1}<B_{1}$ of strata in $W_{1}$ :

Condition D. Let $A_{2}=f\left(A_{1}\right)$ and $B_{2}=f\left(B_{1}\right)$ and suppose $x_{i} \in B_{1}$ converges to a point $y \in A_{1}$. Suppose the tangent planes $T_{x_{i}} B_{1}$ converge to some plane $\tau_{1} \subset$ $T_{y} M_{1}$ and suppose $T_{f\left(x_{i}\right)} B_{2}$ converge to some plane $\tau_{2} \subset T_{f(y)} M_{2}$. Then $d f(y)\left(\tau_{1}\right) \supset$ $\tau_{2}$.

For example, if $K_{1} \rightarrow M_{1}$ and $K_{2} \rightarrow M_{2}$ are smooth triangulations such that the projection $K_{1} \rightarrow K_{2}$ is simplicial, then $f$ satisfies condition $\mathrm{D}$ with respect to the stratifications given by the simplices of $K_{1}$ and $K_{2}$.

LeMma (i). Suppose $\pi: E \rightarrow M_{2}$ is a Riemannian vector bundle and $M_{1}=\{v \in$ $E \mid\langle v, v\rangle=\varepsilon\}$ denotes the $\varepsilon$-sphere bundle of E. Suppose $W_{i} \subset M_{i}$ are Whitney objects such that $\pi$ becomes a stratified submersion satisfying condition $\mathrm{D}$. Then the mapping cylinder

$$
c\left(W_{1}\right)=\left\{t x \mid t \in[0,1], x \in W_{1}\right\}
$$

satisfies the Whitney conditions with respect to its (obvious) canonical stratification.

LEMMA (ii). Suppose furthermore that $f: M_{2} \rightarrow M_{3}$ is a smooth fibration, that $W_{3} \subset M_{3}$ is a Whitney object such that $f$ becomes a stratified submersion which satisfies condition D. Then the composition $f \circ \pi: c\left(W_{1}\right) \rightarrow W_{3}$ is a stratified submersion which satisfies condition D.

Proof. Suppose $S_{1}$ is a stratum of $W_{1}$ and $R_{2}<S_{2}=\pi\left(S_{1}\right)$ are strata of $W_{2}$. We shall show for example, that the pair $R_{2}<c^{\circ}\left(S_{1}\right)$ satisfies Whitney's condition B, where $c^{\circ}\left(S_{1}\right)$ denotes the "open cone" $\left\{t x \mid x \in S_{1}\right.$ and $\left.t \in(0,1)\right\}$. The problem is local so we may assume $E=\mathbf{R}^{n} \times \mathbf{R}^{k} \rightarrow \mathbf{R}^{n} \times\{0\}$ and $M_{1}=\mathbf{R}^{n} \times S^{k-1}(\varepsilon)$. Suppose $p_{i} \in c^{\circ}\left(S_{1}\right)$ and $q_{i} \in R_{2}$ are sequences of points converging to some $q_{0} \in R_{2}$. Suppose the secant lines

$$
l_{i}=\overbrace{p_{i}} q_{i}
$$

converge to some line $l$, and the tangent planes $T_{p_{i}}\left(c^{\circ}\left(S_{1}\right)\right)$ converge to $\tau$. We must show $l \subset \tau$. Now $c^{\circ}\left(S_{1}\right)$ is the image of $S_{1} \times(0,1)$ under the smooth map $G$ : $\mathbf{R}^{n} \times \mathbf{R}^{k} \times[0,1] \rightarrow \mathbf{R}^{n} \times \mathbf{R}^{k}$ given by $G(a, b, t)=(a, t b)$, so we may write $p_{i}=$ $\left(a_{i}, t_{i} b_{i}\right) \rightarrow\left(q_{0}, 0\right)$ and $q_{i}=\left(q_{i}, 0\right) \rightarrow\left(q_{0}, 0\right)$, where $\left(a_{i}, 0\right) \in S_{2}$ and $\left(a_{i}, b_{i}\right) \in S_{1}$. By taking subsequences if necessary we may assume $\left(a_{i}, b_{i}\right)$ converge to some point $\left(q_{0}, b_{0}\right) \in \bar{S}_{1}$. Let $R_{1}$ denote the stratum of $W_{1}$ which contains this point.

A unit vector $\left(v_{0}, w_{0}\right) \in l$ can be obtained as the limit of vectors $\alpha_{i}\left(a_{i}-q_{i}, t_{i} b_{i}\right)$ for an appreciate choice of $\alpha_{i} \in \mathbf{R}$. Then $\alpha_{i}\left(a_{i}-q_{i}, 0\right)$ (which span the secant lines

$$
\left.\left(a_{i}, 0\right),\left(q_{i}, 0\right)\right)
$$


converge to $(v, 0)$. Condition B for $R_{2}<S_{2}$ implies that $\left(v_{0}, 0\right) \in \lim T_{\left(a_{i}, 0\right)} S_{2}$ so by condition $\mathrm{D}$ there is a vector $(v, u) \in \lim T_{\left(a_{i}, b_{i}\right)} S_{1}$ such that $d \pi\left(a_{0}, b_{0}\right)(v, u)=$ $\left(v_{0}, 0\right)$, i.e. $v=v_{0}$. If we take $\left(v_{i}, u_{i}\right) \in T_{\left(a_{i}, b_{i}\right)} S_{1}$ converging to $(v, u)$ then the sequence $d G\left(a_{i}, b_{i}, t_{i}\right)\left(v_{i}, u_{i}, \alpha_{i} t_{i}\right)=\left(v_{i}, t_{i} u_{i}+\alpha_{i} t_{i} b\right)$ lies in $T_{p_{i}} c^{\circ}\left(S^{\prime}\right)$ and converges to $\left(v_{0}, w_{0}\right)$ as desired.

The proof of part (ii) is entirely similar.

A.2. Whitney cellulations of spaces with conical singularities.

Definition. Let $M$ be a manifold containing a Whitney stratified set $X$ with a fixed system of control data. Let $A$ be a stratum of $X$ and (as in $\S 2$ ) let $E_{A}$ denote the Riemannian normal bundle of $A$ in $M$, with embedding $\phi_{A}: E_{A}(\varepsilon) \rightarrow T_{A}(\varepsilon) \subset$ $M$ determined by the control data. Define a radial retraction $r_{A}(\varepsilon): T_{A}-A \rightarrow$ $S_{A}(\varepsilon)$ by

$$
r_{A}(\varepsilon)(p)=\phi\left(\frac{\phi^{-1}(p) \cdot \varepsilon}{\left|\phi^{-1}(p)\right|}\right)
$$

Note that $\pi_{A} r_{A}(\varepsilon)=\pi_{A}$.

A stratum $B>A$ is said to be conical near $A$ (with respect to this tubular neighborhood) if, for some $\varepsilon>0, B \cap T_{A}(\varepsilon)=r_{A}(\varepsilon)^{-1}\left(B \cap S_{A}(\varepsilon)\right)$. The tubular neighborhood $T_{B}$ of such a stratum $B$ is said to be conical if the following commutation relations hold for some $\varepsilon>0$ :

$$
r_{A}(\varepsilon) \circ r_{B}(\varepsilon)=r_{B}(\varepsilon) \circ r_{A}(\varepsilon), \quad \rho_{B} \circ r_{A}(\varepsilon)=\rho_{B} .
$$

(It follows that $\pi_{A} \circ r_{B}(\varepsilon)=\pi_{A}$ and $\rho_{A} \circ r_{B}(\varepsilon)=\rho_{A}$.)

We shall say $X$ is conical near $A$ if each stratum is conical near $A$, and similarly for conical control data near $A . X$ has conical singularities if it is conical near each stratum and admits conical control data.

Proposition. Suppose $X$ is a Whitney object with conical singularities and conical control data. Then there exists a (regular) cell decomposition of $X$ into smooth cells such that the stratification given by the cells satisfies the Whitney conditions.

Proof. For each stratum $A$, let $A_{\varepsilon}^{\circ}$ be the subset of $A$ obtained by removing the open $\varepsilon$-tubular neighborhooods of all strata $B<A$. Thus $A_{\varepsilon}^{\circ}$ is a manifold with corners. Similarly let $S_{A}^{\circ}(\varepsilon)=S_{A}(\varepsilon)-\cup T_{B}(\varepsilon)$, the union taken over all $B<A$. It is proven in [7] that there is a simplicial complex $K$ and a homeomorphism $f$ : $|K| \rightarrow \cup_{\text {all } A} A_{\varepsilon}^{\circ}$ such that $f$ is smooth on each simplex and such that each projection $\pi_{A}^{\circ}: S_{A}^{\circ}(\varepsilon) \rightarrow A_{\varepsilon}^{\circ}$ becomes simplicial. In particular, for each simplex $\sigma \in K$, the closure $f(\bar{\sigma})$ satisfies the Whitney conditions. Therefore a cellulation of $X$ is obtained by (conically) cellulating each of the mapping cylinders of $\pi_{A}^{\circ}$ starting with the largest stratum and working down to the smallest as in [7]. However each projection $\pi_{A}^{\circ}$ satisfies condition D by Lemma A2(ii) and induction. Therefore the conical mapping cylinder satisfies the Whitney conditions by Lemma A2(i).

REMARK. The cells of this conical mapping cylinder do not carry a natural linear structure: they must be subdivided in order to carry a P.L. stucture. I do not know whether the subdivided mapping cylinder of [7] satisfies the Whitney conditions in this context. 


\section{A.3. Deforming a space to one with conical singularities.}

Definition. If $X$ and $X^{\prime}$ are Whitney stratified subsets of a manifold $M$, a deformation of $X$ to $X^{\prime}$ is a Whitney stratified subset $Y \subset M \times[0,1]$ together with a stratum preserving homeomorphism $Y \rightarrow X \times[0,1]$ which is smooth on each stratum of $Y$ and commutes with the projection to [0,1]; such that $Y \cap M \times\{0\}$ $=X \times\{0\}$ and $Y \cap M \times\{1\}=X^{\prime} \times\{1\}$. (If $X$ is the support of a geometric cycle in $M$, it follows that $X$ and $X^{\prime}$ are cobordant.)

Proposition. Let $X$ be a Whitney stratified subset of a manifold $M$. Then $X$ can be deformed to a Whitney object $X^{\prime}$ which has conical singularities.

Proof. By induction, we suppose that $X$ is conical near each stratum of its $n-1$-skeleton and that $X$ has a system of control data which is conical near each stratum of its $n-1$-skeleton. We must deform $X$ until it is conical (and admits conical control data) near the $n$-skeleton.

Fix an $n$-dimensional stratum $A$ of $X$. By a change of scale on the normal bundle $E_{A}$, we may assume there is a tubular neighborhood of radius $2, \phi_{A}: E_{A}(2) \rightarrow T_{A}(2)$ $\subset M$, such that for each $\delta \leqslant 2, S_{A}(\delta)$ is transverse to $X$.

The idea is to replace (at each time $t \in[0,1])$ the portion $X \cap \overline{T_{A}(t)}$ by the mapping cylinder of $X \cap S_{A}(t) \rightarrow A$ defined conically within $E_{A}$. This mapping cylinder is obtained as the pre-image of $S_{A}(t)$ by the radial retraction $r_{A}(t)$ : $T_{A}(t)-A \rightarrow S_{A}(t)$. Then the "kink" which occurs along $X \cap S_{A}(t)$ must be smoothed out.

If $\alpha: \mathbf{R} \rightarrow \mathbf{R}$ is a smooth function such that $\alpha(t) \geqslant 0, \alpha^{\prime}(t) \geqslant 0, \alpha(t)=0$ whenever $t \leqslant 1$, and $\alpha(t)=1$ whenever $t \geqslant 1 \frac{1}{3}$, we can define $F: M \times[0,1] \rightarrow M$ as follows:

$$
F(p, t)=\left\{\begin{array}{l}
p \quad \text { if } p \in \bar{A} \text { or if } p \notin T_{A}(2) \\
\frac{p}{|p|}\left(t+\alpha\left(\frac{|p|}{t}\right)(|p|-t)\right) \text { if } p \in T_{A}(2)-A
\end{array}\right.
$$

We claim $Y=F^{-1}(X) \subset M \times[0,1]$ is the desired deformation.

REMARKs. (1) The maps $\phi_{A}$ and $\phi_{A}^{-1}$ have been surpressed. The scalar multiplications are to be taken fibrewise within the vector bundle structure of $E_{A}$. Similarly $|p|$ denotes $\left\langle\phi_{A}^{-1}(p), \phi_{A}^{-1}(p)\right\rangle^{1 / 2}$.

(2) $F$ is not continuous at $(p, t)$ when $p \in A$.

(3) To aid in the calculations, a specific choice of the function $\alpha$ will be made below.

Let $F_{t}(p)=F(p, t)$. We identify $X$ with $F_{0}^{-1}(X)=Y \cap M \times\{0\}$. The homeomorphism $Y \rightarrow X \times[0,1]$ can be constructed using controlled vector fields as in [11].

Let $X^{\prime}=F_{1}^{-1}(X)$. Then $X^{\prime}$ is conical near $F_{1}^{-1}(A)$ because it coincides with the conical mapping cylinder of $S_{A}(1) \rightarrow A$. In fact, whenever $|p|<t$ we have $F_{t}(p)=$ $r_{A}(t)(p) . X^{\prime}$ is also conical near each stratum $F_{1}^{-1}(B)<F_{1}^{-1}(A)$ since the whole procedure is conical near $B$. 
We wish to verify the Whitney conditions on $X^{\prime}$ and on $Y$. (We shall say the Whitney conditions hold near a stratum $C$ if they hold for every pair $C<D$.) If $\delta \leqslant 2$ then $F_{t}$ maps each sphere bundle $S_{A}(\delta)$ isomorphically to $S_{A}\left(\delta^{\prime}\right)$ (where $\left.\delta^{\prime} \leqslant 2\right)$ which is transverse to $X$. Then $F_{t} \mid(M-\bar{A})$ is transverse to $X$, so $F^{-1}(X)$ (and $F_{1}^{-1}(X)=X^{\prime}$ ) satisfy the Whitney conditions everywhere except (possibly) near $F^{-1}(\bar{A})$ (and $F_{1}^{-1}(\bar{A})$, respectively). However $X^{\prime}$ is conical near $F_{1}^{-1}(A)$ and also near each $F_{1}^{-1}(B)<F_{1}^{-1}(A)$, so Lemma A2(i) guarantees that the Whitney conditions hold there as well. A similar remark applies to $Y$ near the strata $F^{-1}(A) \cap M$ $\times(0,1)$ and $F^{-1}(B) \cap M \times(0,1)$ for all strata $B<A$. However we must still check the Whitney conditions on $Y$ near the strata $F_{0}^{-1}(B)$ and $F_{0}^{-1}(A)$.

In order to calculate the tangent space of each stratum $F^{-1}(C)$ where $C>A$, we parametrize $Y=F^{-1}(X)$ near $F^{-1}(A)$ as the image of $X \times[0,2]$ under a map $G$ : $X \times[0,2] \rightarrow E_{A} \times[0,1]$ which is defined as follows:

Fix a smooth function $h(s) \geqslant 0$ with $h(s)=0$ for $s \leqslant 1, h(s)=1$ for $s \geqslant 1 \frac{1}{4}$, and $h^{\prime}(s) \geqslant 0$ for all $s$. Set $g_{1}(s)=s+(1-s) h(s), g_{2}(s)=1+(1-s) h(s)$, and $g(s)=$ $g_{1}(s) / g_{2}(s)$. Then $g^{\prime}(s) \neq 0$ on $[0,2)$ so $g$ is invertible. Let $\alpha(t)=h\left(g^{-1}(t)\right)$. Use this function $\alpha$ in the definition of $F$ above. If we define $G: E_{A} \times[0,2] \rightarrow E_{A} \times[0,1]$ by $G(p, t)=\left(p \cdot g_{1}(t),|p| g_{2}(t)\right)$ then it is tedious but straightforward to see that

$$
F(G(p, t))=p \quad \text { and } \quad G(F(p, t), m(|p| / t))=(p, t)
$$

where $m(s)=1+(s-1) /(1+(s-1) \alpha(s))$. This shows that for each stratum $C>A$, the stratum $F^{-1}(C)$ coincides with the image $G(C \times[0,2])$ near $F^{-1}(A)$.
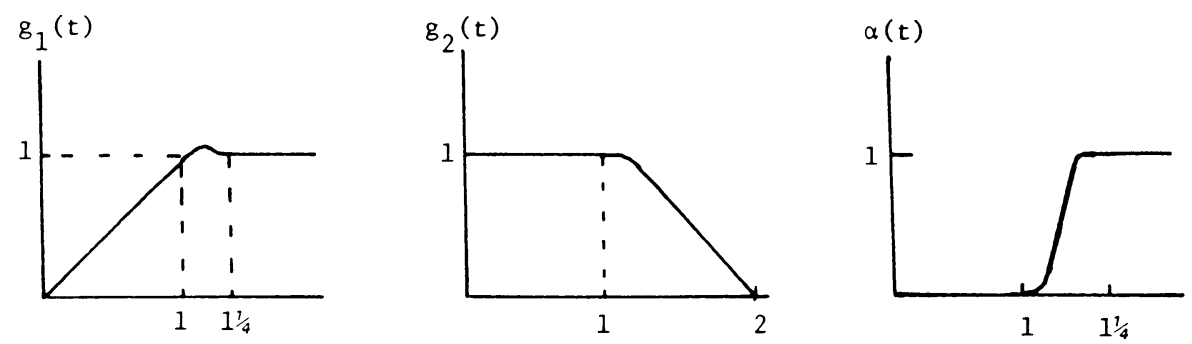

Locally on $A$ the bundle $\pi_{A}: E_{A} \rightarrow A$ may be trivialized to the projection $\mathbf{R}^{n} \times \mathbf{R}^{k} \rightarrow \mathbf{R}^{n}$ where

$$
\pi_{A}\left(x_{1}, \ldots, x_{n+k}\right)=\left(x_{1}, \ldots, x_{n}, 0, \ldots, 0\right)
$$

and

$$
\left|\left(x_{1}, \ldots, x_{n+k}\right)\right|=\left\|\left(x_{k+1}, \ldots, x_{n+k}\right)\right\|=\left(x_{k+1}^{2}+\cdots+x_{k+n}^{2}\right)^{1 / 2} .
$$

In these coordinates, $G$ takes the form

$$
G(x, y, t)=\left(x, y g_{1}(t),\|y\| g_{2}(t)\right) \text {. }
$$

Let $C$ be a stratum of $X$ with $A<C$. We wish to study the Whitney condition for the pair $A<F^{-1}(C) \cap M \times(0,1)$. Suppose the sequence $p_{i}=G\left(x_{i}, y_{i}, t_{i}\right) \in$ $F^{-1}(C)$ converge to a point $\left(x_{0}, 0,0\right) \in A$ and suppose a sequence $\left(x_{i}^{\prime}, 0,0\right) \in A$ also converge to $\left(x_{0}, 0,0\right)$. Suppose the secant lines

$$
(x_{i}^{\prime}, \overbrace{0,0), p_{i}}
$$


converge to a line $l$ and suppose the tangent planes $T_{p_{i}} F^{-1}(C)$ converge to a plane $\tau$. We must show $l \subset \tau$.

It follows that $\left(x_{i}, y_{i}, 0\right) \in C$ converge to $\left(x_{0}, 0,0\right) \in A$ however the $t_{i}$ do not necessarily converge to 0 . A unit vector $\hat{l}$ in $l$ can be obtained as the limit of the vectors

$$
\alpha_{i}\left(x_{i}-x_{i}^{\prime}, y_{i} g_{1}\left(t_{i}\right),\left\|y_{i}\right\| g_{2}\left(t_{i}\right)\right)
$$

for an appropriate choice of $\alpha_{i} \in \mathbf{R}$. By taking subsequences, we may assume the vectors $\alpha_{i}\left(x_{i}-x_{i}^{\prime}, y_{i}, 0\right)$ also converge; thus condition B guarantees that there is a sequence of vectors $\left(a_{i}, b_{i}, 0\right) \in T_{\left(x_{i} y_{i}, 0\right)} C$ approaching the same limit. It now follows by direct calculation that the vectors

$$
d G\left(x_{i}, y_{i}, t_{i}\right)\left(a_{i}, b_{i}, 0\right) \in T_{p_{i}} F^{-1}(C)
$$

converge to the vector $\hat{l}$, completing the verification of Whitney's condition $\mathbf{B}$ in this case.

Similarly if $B<A<C$, we must verify the Whitney conditions for the incidence $B<F^{-1}(C) \cap M \times(0,1)$. However, the whole construction is conical near $B$, so it suffices to check that the projection $F^{-1}\left(S_{B}(\varepsilon)\right) \rightarrow F^{-1}(B)$ satisfies condition D of A2. This verification is entirely similar to the preceding analysis and will be omitted.

To complete the induction we must construct control data on $X^{\prime}$ which is conical near $A$ and near each stratum $B<A$. This involves adjusting only the tubular neighborhoods $T_{C}$ for strata $C>A$. However, the procedure of Thom and Mather [13] does exactly that: one simply observes that if the space $X^{\prime}$ is conical near $B$ then the control data (which is constructed by coning down to $B$ control data previously defined on $S_{B}(\varepsilon)$ ) is also conical near $B$.

Relative version of Proposition A.3. Suppose $M$ is a manifold with collared boundary (or even a manifold with collared corners) and suppose $X \subset M$ is a Whitney object which respects the collaring. Then the whole procedure may be applied first to $X \cap \partial M$, then extended to the interior of $M$ using the collar, then extended to all of $M$ using a partition of unity, giving a (collar-preserving) deformation of $X$ to a Whitney object $X^{\prime}$ which has conical singularities.

\section{REFERENCES}

1. S. Buoncristiano, C. Rourke and B. Sanderson, A geometric approach to homology theory, London Math. Soc. Lecure Notes, vol. 18, Cambridge Univ. Press, New York, 1976.

2. A. Dold and R. Thom, Quasifaserungen und symmetrische produkte, Ann. of Math. (2) 67 (1958), 239-281.

3. H. Federer, Geometric measure theory, Springer-Verlag, New York, 1969.

4. W. Fulton and R. MacPherson, Categorical framework for the study of singular spaces, Mem. Amer. Math. Soc., no. 243, 1981.

5. Gibson et al., Topological stability of smooth mappings, Lecture Notes in Math., vol. 552, SpringerVerlag, Berlin and New York, 1976.

6. M. Golubitsky and V. Guillemin, Stable mappings and their singularities, Graduate Texts in Mathematics, no. 14 , Springer-Verlag, Berlin and New York, 1973.

7. M. Goresky, Triangulation of stratified objects, Proc. Amer. Math. Soc. 72 (1978), 193-200.

8. M. Goresky and R. MacPherson, Intersection homology theory, Topology 19 (1980), 135-162.

9. R. Hardt, Homology theory for real analytic and semi-analytic sets, Ann. Scuola Norm. Sup. Pisa Cl. Sci. (4) 2 (1975), 107-148. 
10. __ Topological properties of subanalytic sets, Trans. Amer. Math. Soc. 211 (1975), 57-70.

11. C. McCrory, Poincare duality in spaces with singularities, Thesis, Brandeis University, 1972.

12. R. MacPherson, "Generic vector bundle maps" in Dynamical systems edited by M. M. Peixoto, Academic Press, New York, 1973.

13. J. Mather, Notes on topological stability, Mimeographed notes, Harvard University, 1970.

14. , "Stratifications and mappings" in Dynamical systems edited by M. M. Peixoto, Academic

Press, New York, 1973.

15. J. Milnor and J. Stasheff, Characteristic classes, Ann. of Math. Studies, no. 76, Princeton Univ. Press, Princeton, N.J., 1974.

16. J. Milnor, Morse theory, Ann. of Math. Studies, no. 51, Princeton Univ. Press, Princeton, N.J., 1963.

17. D. Sullivan, “A general problem" (in Manifolds, Amsterdam, 1970), Lecture Notes in Math., vol. 197, Springer-Verlag, Berlin and New York, 1971.

18. __ "Singularities in spaces" (in Proceedings of Liverpool Singularities, Symposium. II), Lecture Notes in Math., vol. 209, Springer-Verlag, Berlin and New York, 1971.

19. R. Thom, Quelques propriétés globales des variétés différentiables, Comment. Math. Helv. 28 (1954), $17-86$.

20. ___ Ensembles et morphismes stratifiés, Bull. Amer. Math. Soc. 75 (1969), 240-284.

21. __ "Problems in the study of singularities" in Mathematical developments arising from Hilbert problems, Proc. Sympos. Pure Math., vol. 28, Part 1, Amer. Math. Soc., Providence, R.I., 1976, p. 58.

22. A. Verona, Integration on Whitney prestratifications, Rev. Roumaine Math. Pures Appl. 17 (1972), 1473-1480.

23. __ Homological properties of abstract prestratifications, Rev. Roumaine Math. Pures Appl. 17 (1972), 1109-1121.

24. G. W. Whitehead, Generalized homology theories, Trans. Amer. Math. Soc. 102 (1962), 227-283.

25. H. Whitney, Geometric methods in cohomology, Proc. Nat. Acad. Sci. U.S.A. 33 (1947), 7-9.

Department of Mathematics, University of British Columbia, Vancouver, Brtish Columbia, CANADA V6T 1 Y4 